\title{
Optimum Material Gradient for Functionally Graded Rectangular Plate with the Finite Element Method
}

\author{
Wasim M. K. Helal and Dongyan Shi \\ College of Mechanical \& Electrical Engineering, Harbin Engineering University, Harbin 150001, China \\ Correspondence should be addressed to Wasim M. K. Helal; dr_waseemhelal@eng.kfs.edu.eg
}

Received 7 October 2013; Accepted 16 December 2013; Published 17 February 2014

Academic Editors: V. Rajagopal Reddy and V. Srivastava

Copyright ( 2014 W. M. K. Helal and D. Shi. This is an open access article distributed under the Creative Commons Attribution License, which permits unrestricted use, distribution, and reproduction in any medium, provided the original work is properly cited.

The optimum material gradient of a rectangular plate made of functionally graded material (FGM) is determined in this study. Elastic modulus of functionally graded (FG) rectangular plate is assumed to vary continuously throughout the height of the plate, according to the volume fraction of the constituent materials based on the power law, exponential model I, exponential model $\Pi$, or sigmoid functions. The difference between these distribution functions for the constituents' volume fraction is discussed in this study. To determine the optimum material gradient of a rectangular plate made of FGM, the finite element method and the optimization techniques are used. In this study, von Mises stress, shear stress, and deformation in FGM case with the power law, exponential model I, exponential model $\Pi$, or sigmoid functions are investigated. Simulation results indicate that the optimum material gradient for FG rectangular plate can be described by using a modified sigmoid function. The maximum values of von Mises stress, shear stress, and deformation in FG rectangular plate with the optimum material gradient are reduced compared with the pure material case by around $22 \%, 11 \%$, and $24 \%$, respectively.

\section{Introduction}

Because of the demand of conflicting property requirement in engineering applications, pure metals are of little use. To satisfy the high performance of industrial demands and to eliminate the stress singularities (Hirano and Yamada [1]; Niino and Maeda [2]), one way to achieve these objectives is to use "functionally graded materials (FGMs)" $[3,4]$.

Before FGMs were found, over the past three decades in Japan thin layers or laminates were used in plane structures, but temperature variation occurred through the thickness. This temperature variation could produce thermal deformation and the thin layers separated, so engineers decided to find a new idea using different materials or a new method to avoid the cause of separation. After engineers did some experimental tests, a new material was developed, which was defined as FGM. FGMs are microscopically inhomogeneous in which the mechanical properties vary smoothly and continuously from one surface to the other $[5,6]$.

Power law function (Yung and Munz [7]; Jin and Paulino [8]) and exponential function (Delale and Erdoogan [9], Jin and Noda [10], Jin and Batra [11], Erdogan and Wu [12], Gu and Asaro [13], and Erdogan and Chen [14]) are commonly used to describe the material properties variations of FGMs. A stress concentration appears due to abrupt changes of the distribution for the volume fractions in both power law and exponential functions. Chung and Chi [15] proposed a sigmoid function to define a new distribution for the volume fraction which can be used to reduce the stress intensity factor (Chi and Chung [16]).

The primary objective of this study is to discuss the difference between these distribution functions for the constituents' volume fraction and using the optimization techniques that are available in the ANSYS package [17] to determine the optimum material gradient of a rectangular plate made of FGM. Very few investigators have studied the difference between the power law, exponential, or sigmoid functions for the constituents' volume fraction. To the best of the author's knowledge, there are no references available in the literature which deal with the determination of the optimum material gradient of a rectangular plate made of FGM.

The present investigation deals with this problem itself. In this study, a two-dimensional case where a rectangular plate is 


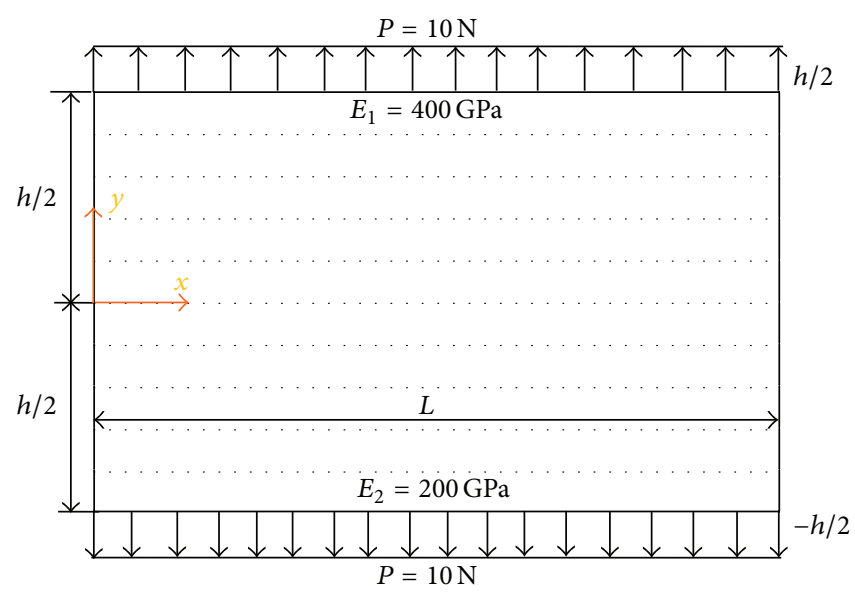

(a)

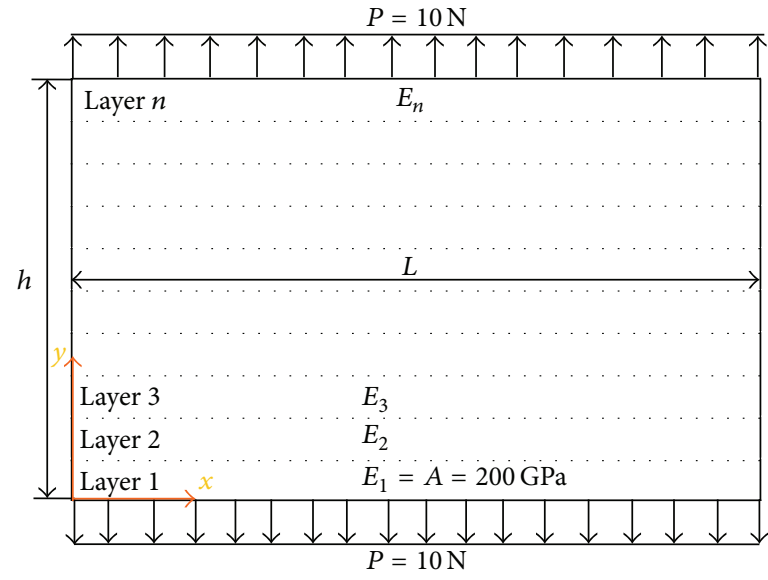

(b)

FIGURE 1: FG rectangular plate of (a) P-FGM, E-FGM model I, and S-FGM cases and (b) E-FGM model П case.

put under uniaxial tension with the two unloaded edges held fixed is considered. Elastic modulus of a rectangular plate is assumed to vary continuously throughout the height of the plate, according to the volume fraction of the constituent materials based on the power law, exponential, or sigmoid functions. Delale and Erdoogan [9] indicated that the effect of Poisson coefficient on the deformation is much less than that of Young's modulus. Thus, the value of Poisson coefficient is constant and was set to 0.3 in this study.

\section{Material Gradient of FGM Plates}

The material properties of FGMs vary smoothly from one surface to another. The power law function, exponential function model I, exponential function model $\Pi$, or sigmoid function are used by most researchers to describe the volume fraction. In this paper, a rectangular plate made of FGM with all previous functions will be considered. Let us consider the case of a rectangular plate of length $L$ and total height $h$ with coordinate system as shown in Figure 1. Figure 1(a) shows the plate of the power law, exponential function model I, and sigmoid function cases. Figure 1(b) shows the plate of the exponential function model $\Pi$ case. In the present work, elastic modulus of FG rectangular plate is assumed to vary according to power law, exponential function model I, or sigmoid function. The values of elastic modulus at $y=-h / 2$ and at $y=h / 2$ are $E_{2}$ and $E_{1}$, respectively, as shown in Figure 1(a). Also, elastic modulus of FG rectangular plate is assumed to vary according to exponential function model $\Pi$. In this case, the material properties vary over the plate from $E_{1}=A=200 \mathrm{GPa}$ to $E_{n}$, as shown in Figure 1(b).

2.1. Characteristics of the Power (P-FGMs) Plates. The volume fraction $\Psi(y)$ of the power (P-FGM) is assumed to obey the following power function:

$$
\Psi(y)=\left(\frac{y+h / 2}{h}\right)^{w}
$$

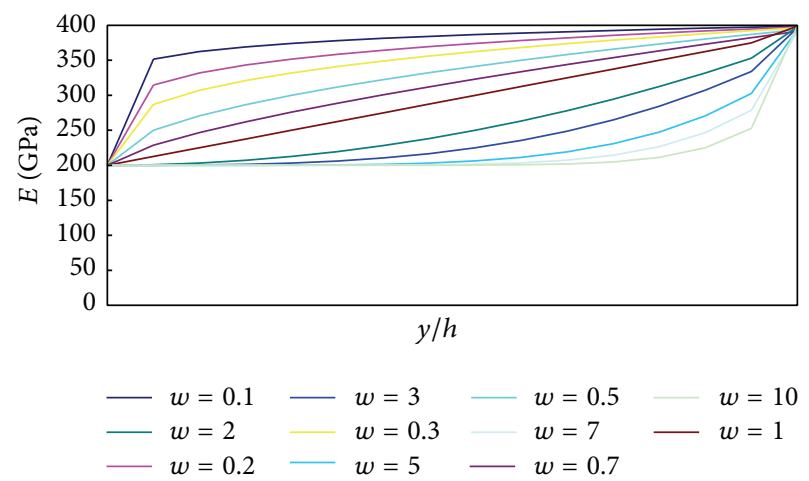

Figure 2: The variation of Young's modulus in a P-FGM plate.

where " $w$ " is the material gradient index and " $h$ " is the height of the plate. After the volume fraction $\Psi(y)$ of the power (PFGM) is defined, the material properties can be determined by the rule of mixture as follows:

$$
E(y)=\Psi(y) E_{1}+[1-\Psi(y)] E_{2},
$$

where $E_{1}$ and $E_{2}$ are the elastic modulus at $(y=h / 2)$ and at $(y=-h / 2)$, respectively. The variation of Young's modulus in the height direction ( $y$-direction) of the power (P-FGM) plate is shown in Figure 2. Figure 2 shows that near $y=-h / 2$ for $w>1$ the elastic modulus changes rapidly and near $y=h / 2$ for $w<1$ increases quickly.

2.2. Characteristics of the Exponential (E-FGM) Plate Model $I$. The exponential function is used by many researchers to describe the variation in elastic modulus as follows [9]:

$$
E(y)=A e^{B(y+h / 2)},
$$

where $A=E_{2}$ and $B=(1 / h) \ln \left(E_{1} / E_{2}\right)$. 


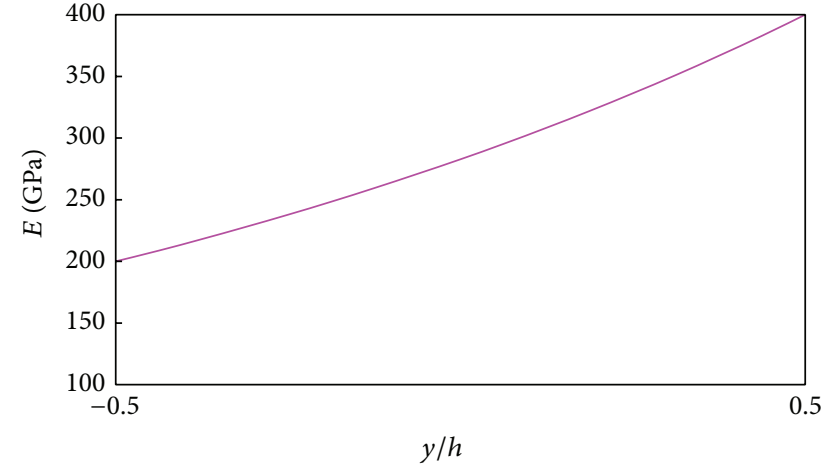

FIGURE 3: The variation of Young's modulus in an E-FGM model I.

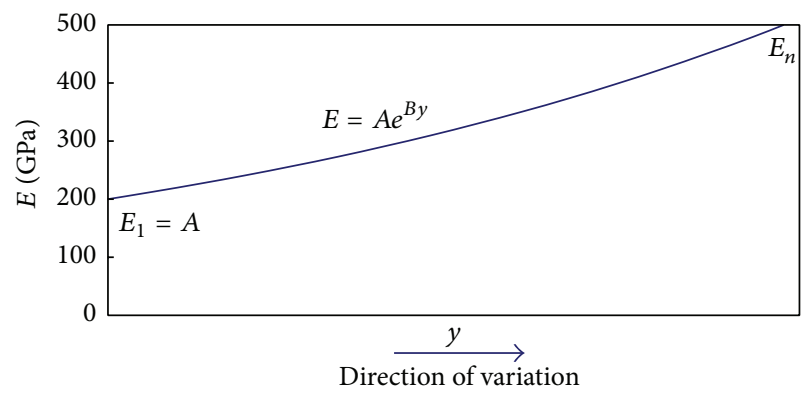

FIGURE 4: The variation of Young's modulus in an E-FGM model П.

The variation in $E(y)$ in the height direction ( $y$-direction) of the (E-FGM) plate model I is shown in Figure 3.

2.3. Characteristics of the Exponential (E-FGM) Plate Model $\Pi$. The variation in elastic modulus for the exponential (EFGM) plate model $\Pi$ case can be defined by the following expression:

$$
E(y)=A e^{B y},
$$

where $A$ is the initial modulus of elasticity of FGM and $B$ is the coefficient of graded in modulus of elasticity of FGM.

In the present work, the value of initial modulus of elasticity of FGM was set to $200 \mathrm{GPa}$ and the value of Poisson's ratio was set to 0.3. In the exponential (E-FGM) plate model $\Pi$, the material properties vary over the plate from $E_{1}=$ $A=200 \mathrm{GPa}$ to $E_{n}$. Figure 4 shows the variation of elastic modulus in the height direction ( $y$-direction) of the (E-FGM) plate model $\Pi$.

2.4. Characteristics of the Sigmoid (S-FGMs) Plate. The stress concentrations appear due to abrupt changes of the distribution for the volume fractions in both power law and exponential functions. Chung and Chi [15] proposed a sigmoid function to define a new distribution for the volume fraction which can be used to reduce the stress intensity factor and to ensure smooth distribution of stresses among all the interfaces (Chi and Chung [16]). The behavior of a

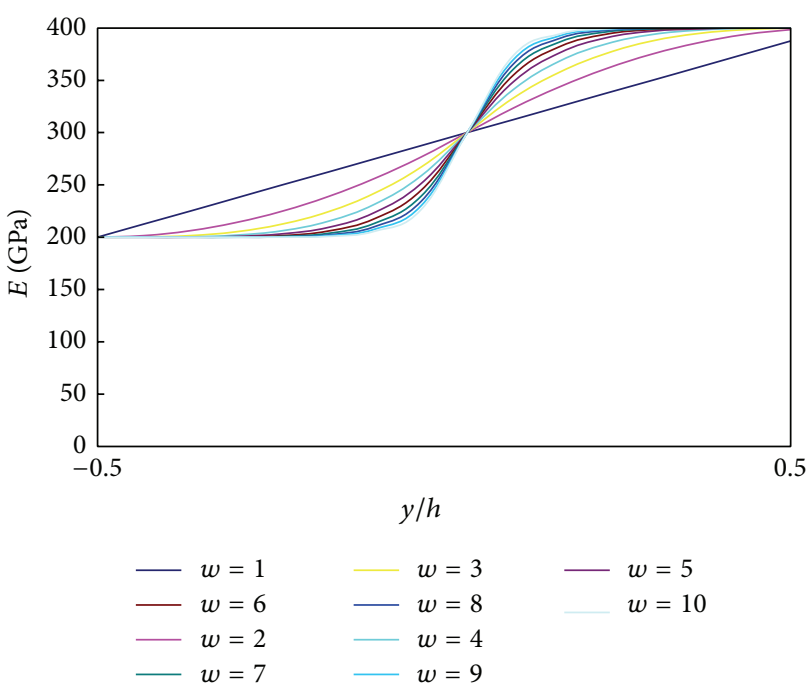

FIgURE 5: The variation of Young's modulus in an S-FGM plate.

sigmoid function is defined by two power law functions as follows:

$$
\begin{gathered}
\Psi_{1}(y)=1-\frac{1}{2}\left(\frac{h / 2-y}{h / 2}\right)^{w} \\
\text { for } 0 \leq y \leq \frac{h}{2} \\
\Psi_{2}(y)=\frac{1}{2}\left(\frac{h / 2+y}{h / 2}\right)^{w} \\
\text { for }-\frac{h}{2} \leq y \leq 0 .
\end{gathered}
$$

By using the rule of mixture, the elastic modulus of the sigmoid (S-FGM) can be evaluated as follows:

$$
\begin{gathered}
E(y)=\Psi_{1}(y) E_{1}+\left[1-\Psi_{1}(y)\right] E_{2} \\
\text { for } 0 \leq y \leq \frac{h}{2}, \\
E(y)=\Psi_{2}(y) E_{1}+\left[1-\Psi_{2}(y)\right] E_{2} \\
\text { for }-\frac{h}{2} \leq y \leq 0 .
\end{gathered}
$$

Figure 5 shows that the variation of elastic modulus is given by (7) and (8) which represent the sigmoid distribution.

\section{Finite Element Analysis and Optimization Technique}

The finite element analysis and optimization were performed using the ANSYS package [17]. In this study, the model was built in ANSYS Rel.12.1 by means of the Parametric Design Language (APDL) to easily manage the properties of the FGM. To model the FGM in the finite element software, the model is divided to a number of homogeneous materials in which the material properties vary due to power law, exponential function model I, exponential function model $\Pi$, 
or sigmoid function. A computer program is developed using ANSYS Parametric Design Language (APDL) to calculate the elastic modulus for power law function, exponential function model $I$, exponential function model $\Pi$, and sigmoid function cases using equations from (1) to (8). Plane elements (PLANE 182) were used to generate the model in $x$ - $y$ plane.

The discrete size of the mesh is $0.1 \mathrm{~mm}$. Sensitivity analysis of a mesh size is carried out. The mesh with discrete size of $0.5,0.3$, and $0.1 \mathrm{~mm}$, respectively, is applied for a rectangular plate model. It is found that the meshes with 0.3 and $0.1 \mathrm{~mm}$ discrete size yield almost identical results, and the maximum von Mises derived from the mesh of $0.3 \mathrm{~mm}$ discrete size has a discrepancy of $0.3 \%$ to that with $0.1 \mathrm{~mm}$ discrete size. On the other hand, with $0.5 \mathrm{~mm}$ discrete size the mesh has substantial difference in terms of von Mises to those from meshes with 0.1 and $0.3 \mathrm{~mm}$ discrete size. Thus, the present work adopts the mesh with a discrete size of $0.1 \mathrm{~mm}$. The final model constituted 16000 elements and 16281 nodes. In this study, a rectangular plate is put under uniaxial tension of a magnitude of $10 \mathrm{~N}$ with the two unloaded edges held fixed, as shown before in Figure 1.

The primary objective of this study is to discuss the differences between the distribution functions for the constituents' volume fraction. After that, the procedure of optimization technique was applied in combination with the above FE model using the ANSYS FE program in order to optimize the material gradient of the FG rectangular plate. The design objective is to minimize the maximum von Mises stress in the plate model. The design variable for this problem is the volume fraction $\Psi(y)$ of the power (P-FGM) and the sigmoid (S-FGM), which is described by the material gradient index " $w$." Thus, the objective function for this problem is to minimize the maximum von Mises stress in a rectangular plate model which is put under uniaxial tension with the two unloaded edges held fixed. The constrains for this problem are

(i) to maintain the value of the design variable " $w$ " within the limits used in the literature $[18,19]$ as $0 \leq w \leq 10$;

(ii) to maintain the maximum shear stress in the plate model less than or equal to the initial value obtained for pure material $(E=200 \mathrm{GPa})$;

$$
\tau_{P} \geq \tau_{\mathrm{FGM}}
$$

where $\tau_{p}$ is the maximum shear stress which is obtained for pure material and $\tau_{\mathrm{FGM}}$ is the maximum shear stress which is obtained for FGM.

\section{Results and Discussion}

The primary objective of this study is to discuss the differences between the distribution functions for the constituents' volume fraction. After that, the procedure of optimization technique was applied using the ANSYS FE program in order to optimize the material gradient of the FG rectangular plate. In this section the responses of P-FGM and S-FGM plates for different values of " $w$ " are compared but the E-FGM plate model I and E-FGM plate model $\Pi$ are compared to the other two functions for $w=1$ because of their characteristics of being independent of " $w$ " [20]. In the final of this section, optimal material gradient for FG rectangular plate is carried out.

4.1. Comparison of the Responses of P-FGM and S-FGM Plates for Different Values of " $w$ ". In the P-FGMs case for small " $w$," the plate will be rich in material, which has a large Young's modulus, and as a result its maximum values of von Mises stress, shear stress, and deformation will be small. However, for large " $w$," the plate will be rich in material, which has a small Young's modulus, and its maximum values of von Mises stress, shear stress, and deformation will be larger. Simulation cases of P-FGM plates for different values of " $w$ " are shown in Figure 6. The results show that the best value of the material gradient index " $w$ " is 0.1 . With $w=0.1$, the maximum values of von Mises stress, shear stress, and deformation equal to 40.378 MPa, $16.308 \mathrm{MPa}$, and 0.000122 , respectively.

On the other hand, simulation cases of S-FGM plates for different values of " $w$ " are shown in Figure 7. The results show that the best value of the material gradient index " $w$ " is 8 . With $w=8$, the maximum values of von Mises stress, shear stress, and deformation equal to $41.356 \mathrm{MPa}, 16.561 \mathrm{MPa}$, and 0.000176 , respectively.

4.2. Comparison of the Responses of P-FGM, E-FGM Model I, E-FGM Model $\Pi$, and S-FGM . The responses of E-FGM model I and E-FGM model $\Pi$ are compared to the other two functions P-FGM and S-FGM for $w=1$ because of their characteristics of being independent of " $w$ " [20]. Figure 8 shows the simulation cases of P-FGM, E-FGM model I, EFGM model $\Pi$, and S-FGM responses in $w=1$. The results show that the minimum values of maximum von Mises stress, shear stress, and deformation are found in the P-FGM and S-FGM cases. As shown in Figure 8 the minimum values of maximum von Mises stress, shear stress, and deformation equal to $42.194 \mathrm{MPa}, 16.812 \mathrm{MPa}$, and 0.000156 , respectively.

4.3. The Optimum Material Gradient. In the present work, the procedure of optimization was applied using ANSYS FE program in order to optimize the material gradient of the FG rectangular plate. In the case of P-FGM, the optimum value of material gradient index " $w$ " equals 0.049685946 . In this case the maximum values of von Mises stress, shear stress, and deformation equal to $40.2792498 \mathrm{MPa}, 16.28 \mathrm{MPa}$, and 0.000109 , respectively. On the other hand, in the case of SFGM, (5) and (6) are modified and become

$$
\begin{gathered}
\Psi_{1}(y)=1-\frac{1}{2}\left(\frac{h / 2-y}{h / 2}\right)^{w} \\
\text { for } 0 \leq y \leq \frac{h}{2}, \\
\Psi_{2}(y)=\frac{1}{2}\left(\frac{h / 2+y}{h / 2}\right)^{D} \\
\text { for }-\frac{h}{2} \leq y \leq 0,
\end{gathered}
$$




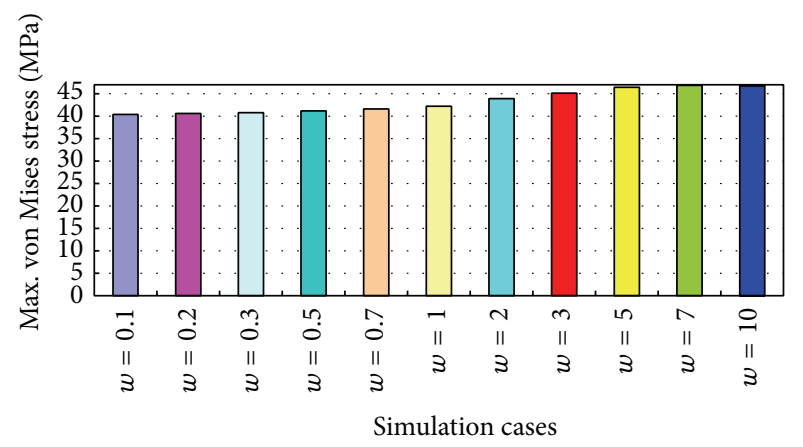

(a)

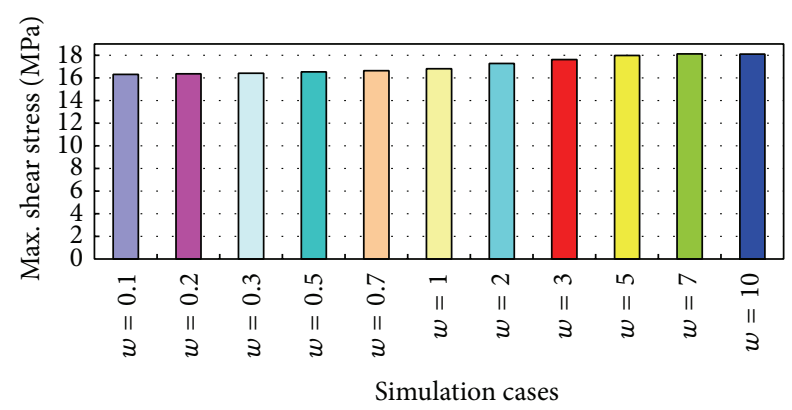

(b)

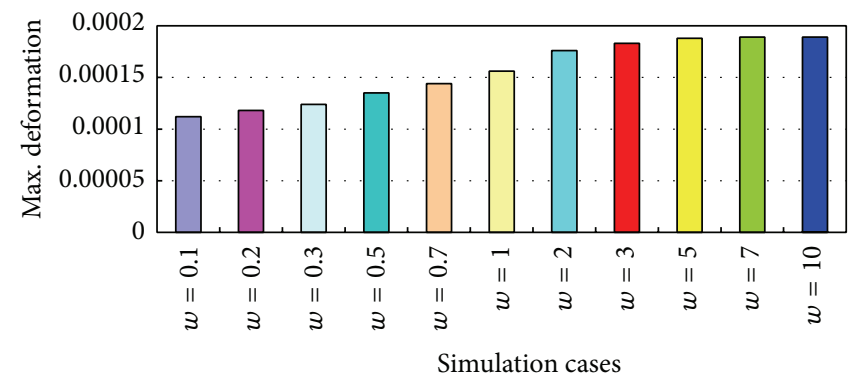

(c)

Figure 6: Simulation cases of P-FGM plates for different values of " $w$ " (a) maximum von Mises stress, (b) maximum shear stress, and (c) maximum deformation.

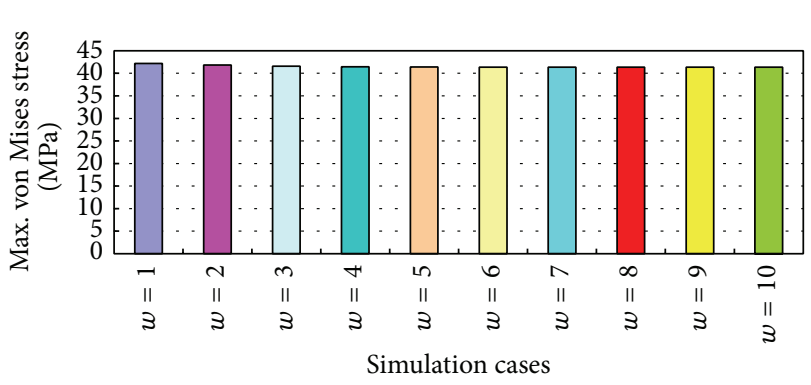

(a)

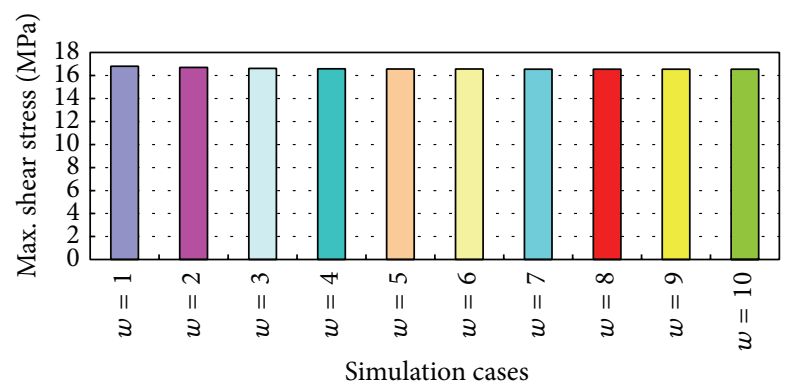

(b)

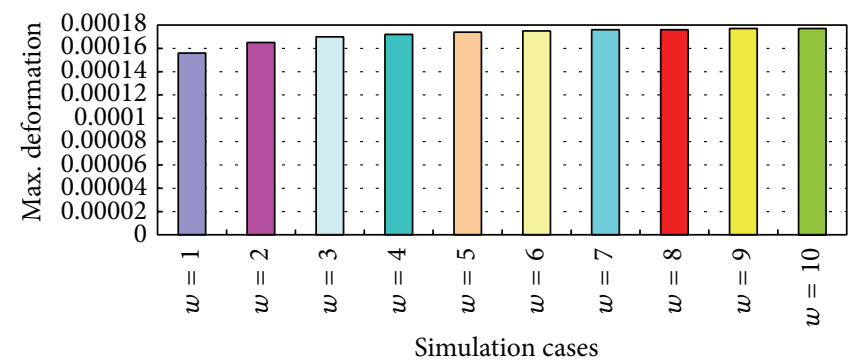

(c)

FIGURE 7: Simulation cases of S-FGM plates for different values of " $w$ " (a) maximum von Mises stress, (b) maximum shear stress, and (c) maximum deformation.

where the optimum values of the material gradient indexes " $w$ " and " $D$ " equal to 0.34116 and 9.1872 , respectively. In this case, the maximum values of von Mises stress, shear stress, and deformation equal to $40.872 \mathrm{MPa}, 16.436 \mathrm{MPa}$, and 0.000141 , respectively. For two cases P-FGM and
S-FGM, Figure 9 shows the optimum material gradient of FG rectangular plate.

Although the results show that the maximum values of von Mises stress, shear stress, and deformation in the P-FGM case are less than in the S-FGM case, the material 


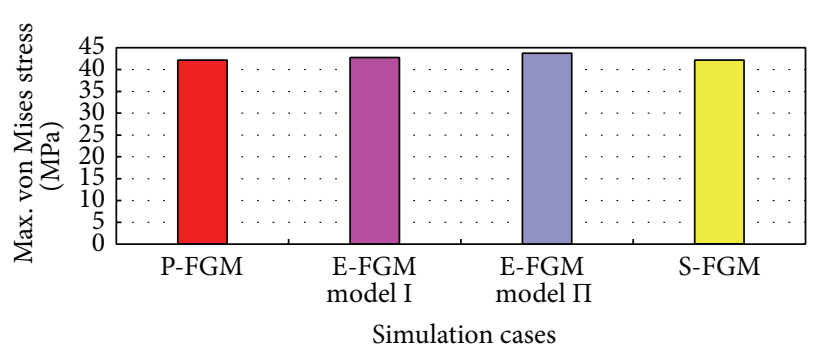

(a)

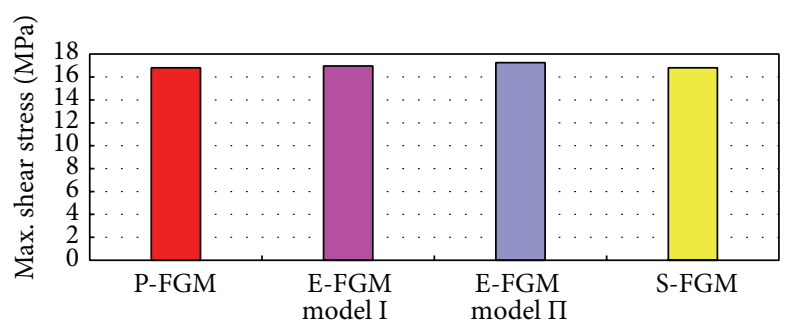

Simulation cases

(b)

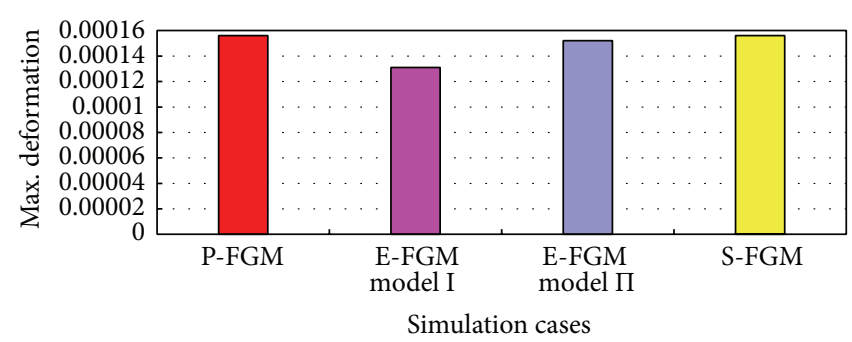

(c)

Figure 8: Comparison of P-FGM, E-FGM model I, E-FGM model П, and S-FGM responses in $w=1$ (a) maximum von Mises stress, (b) maximum shear stress, and (c) maximum deformation.

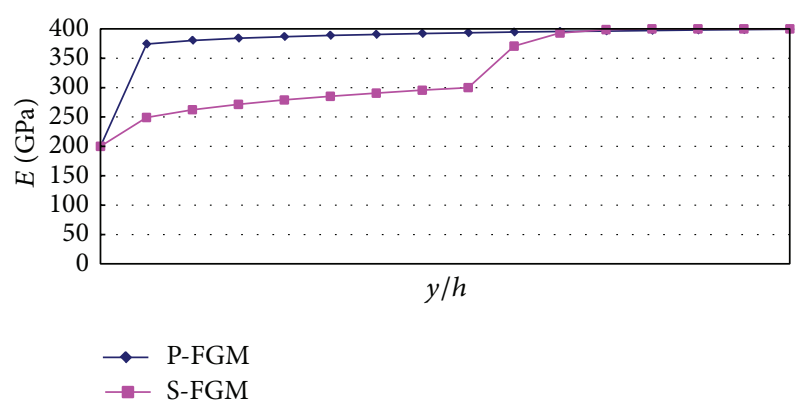

FIGURE 9: The optimum material gradient of FG rectangular plate.

distributions as shown in Figure 9 show that the first step of the variation of Young's modulus in the P-FGM case changed abruptly from $200 \mathrm{GPa}$ to $374 \mathrm{GPa}$. So the optimum material gradient of FG rectangular plate can be descried by using (10) and (11), putting the values of material gradient indexes " $w$ " and " $D$ " equal to 0.34116 and 9.1872 , respectively.

\section{Conclusion}

The following observations can be made based on the present work.

(1) Simulation cases of P-FGM plates for different values of " $w$ " are investigated, and the best value of material gradient index " $w$ " is 0.1 .

(2) Simulation cases of S-FGM plates for different values of " $w$ " are investigated, and the best value of material gradient index " $w$ " is 8 .
(3) Simulation cases of P-FGM, E-FGM model I, E-FGM model $\Pi$, and S-FGM in $w=1$ are investigated, and the minimum values of maximum von Mises stress, shear stress, and deformation are found in the P-FGM and S-FGM cases.

(4) The procedure of optimization technique was applied. The optimum material gradient of FG rectangular plate can be described by using (10) and (11), putting the values of material gradient indexes " $w$ " and " $D$ " equal to 0.34116 and 9.1872, respectively.

(5) The maximum values of von Mises stress, shear stress, and deformation in FG rectangular plate with the optimum material gradient are reduced compared with the pure material case by around $22 \%, 11 \%$, and $24 \%$, respectively.

\section{Conflict of Interests}

The authors declare that there is no conflict of interests regarding the publication of this paper.

\section{Acknowledgments}

The authors thank Kafr El-Sheikh University, Egypt, and Harbin Engineering University, China, for providing the facilities.

\section{References}

[1] T. Hirano and T. Yamada, "Multi-paradigm expert system architecture based upon the inverse design concept," in Proceedings of the International Workshop on Artificial Intelligence for Industrial Applications, pp. 25-27, Hitachi, Japan, 1988. 
[2] M. Niino and S. Maeda, "Recent development status of functionally gradient materials," ISIJ International, vol. 30, no. 9, pp. 699-703, 1990.

[3] M. Yamanoushi, M. Koizumi, T. Hiraii, and I. Shiota, Eds., Proceedings of the 1st International Symposium on Functionally Gradient Materials, Sendai, Japan, 1990.

[4] M. Koizumi, "The concept of FGM. Ceramic transactions," Functionally Gradient Materials, vol. 34, pp. 3-10, 1993.

[5] M. Yamanoushi and M. Koizumi, "Functionally gradient materials," in Proceedings of the 1st International Symposium on Functionally Graded Materials, Sendai, Japan, 1991.

[6] Y. Fukui, "Fundamental investigation of functionally gradient material manufacturing system using centrifugal force," JSME International Journal, vol. 34, no. 1, pp. 144-148, 1991.

[7] Y. Y. Yung and D. Munz, "Stress analysis in a two materials joint with a functionally graded material," in Functionally Graded Material, T. Shiota and M. Y. Miyamoto, Eds., pp. 41-46, 1996.

[8] Z.-H. Jin and G. H. Paulino, "Transient thermal stress analysis of an edge crack in a functionally graded material," International Journal of Fracture, vol. 107, no. 1, pp. 73-98, 2001.

[9] F. Delale and F. Erdogan, "The crack problem for a nonhomogeneous plane," ASME Journal of Applied Mechanics, vol. 50, no. 3, pp. 609-614, 1983.

[10] Z.-H. Jin and N. Noda, "Crack-tip singular fields in nonhomogeneous materials," ASME Journal of Applied Mechanics, vol. 61, no. 3, pp. 738-740, 1994.

[11] Z.-H. Jin and R. C. Batra, "Stress intensity relaxation at the tip of an edge crack in a functionally graded material subjected to a thermal shock," Journal of Thermal Stresses, vol. 19, no. 4, pp. 317-339, 1996.

[12] F. Erdogan and B. H. Wu, "Crack problems in FGM layers under thermal stresses," Journal of Thermal Stresses, vol. 19, no. 3, pp. 237-265, 1996.

[13] P. Gu and R. J. Asaro, "Crack deflection in functionally graded materials," International Journal of Solids and Structures, vol. 34, no. 24, pp. 3085-3098, 1997.

[14] F. Erdogan and Y. F. Chen, "Interfacial cracking of FGM/metal bonds," in Ceramic Coating, K. Kokini, Ed., pp. 29-37, 1998.

[15] Y. L. Chung and S. H. Chi, "The residual stress of functionally graded materials," Journal of the Chinese Institute of Civil and Hydraulic Engineering, vol. 13, pp. 1-9, 2001.

[16] S. H. Chi and Y. L. Chung, "Cracking in sigmoid functionally graded coating," Journal of Mechanics, vol. 18, pp. 41-53, 2002.

[17] ANSYS User's Manual, Version 12.1.

[18] T. Fuchiyama, N. Noda, T. Tsuji, and Y. Obata, "Analysis of thermal stress and stress intensity factor of functionally gradient materials," in Ceramic Transactions: Functionally Gradient Materials, I. B. Holt, Ed., vol. 34, pp. 425-432, American Ceramic Society, Westerville, Ohio, USA, 1993.

[19] T. Fuchiyama and N. Noda, "Multiple crack growths in the functionally graded plate under thermal shock," in Proceedings of the 4th International Congress on Thermal Stresses, pp. 121-124, Osaka, Japan, June 2001.

[20] H. Dastoom Laatleyli and A. Abedian, "A study on the axial stresses of P-FGM, S-FGM and E-FGM plates under pressure loading using the energy concept," in Proceedings of the 27th International Congress of the Aeronautical Sciences, pp. 19-24, Nice, France, September 2010. 

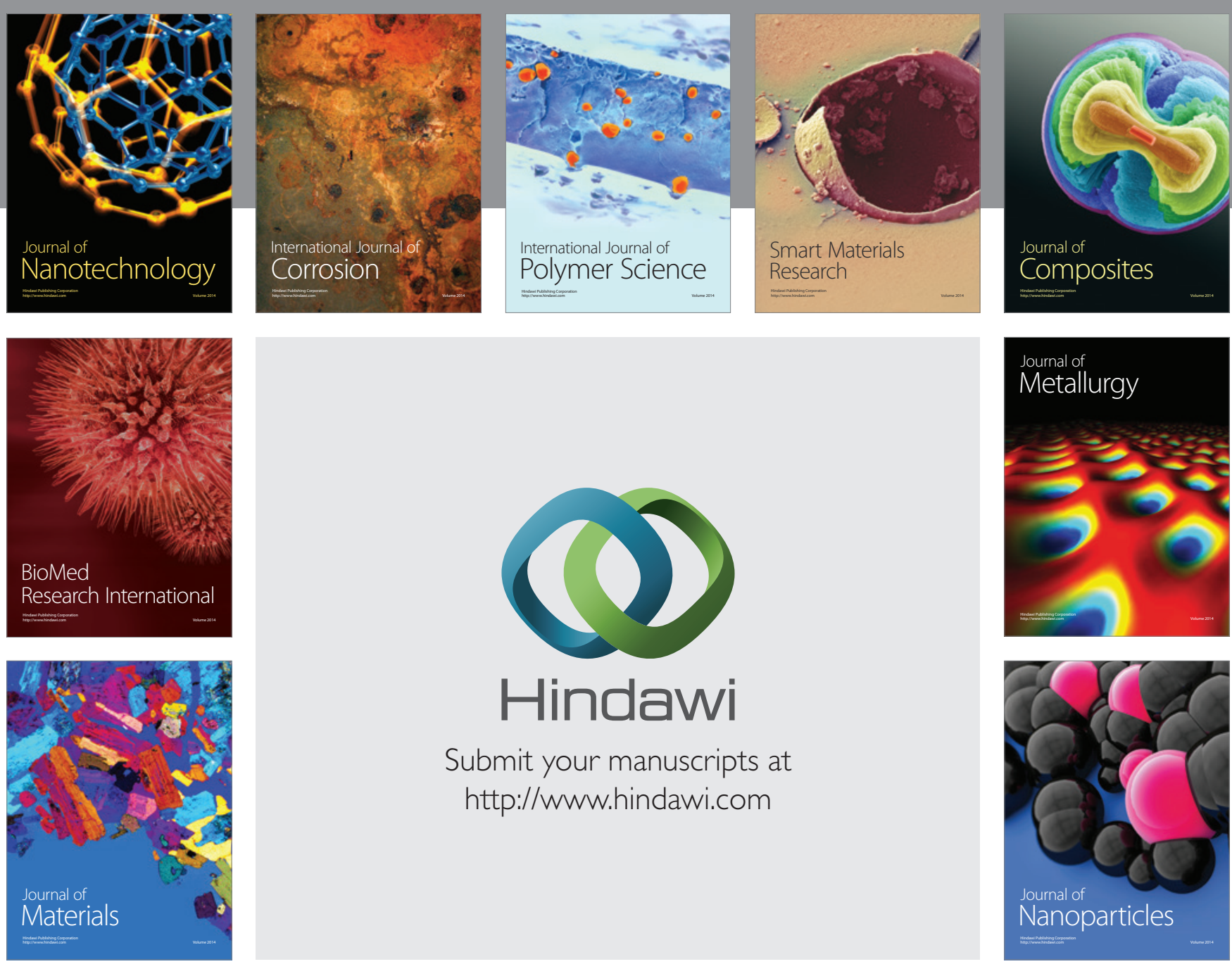

Submit your manuscripts at http://www.hindawi.com
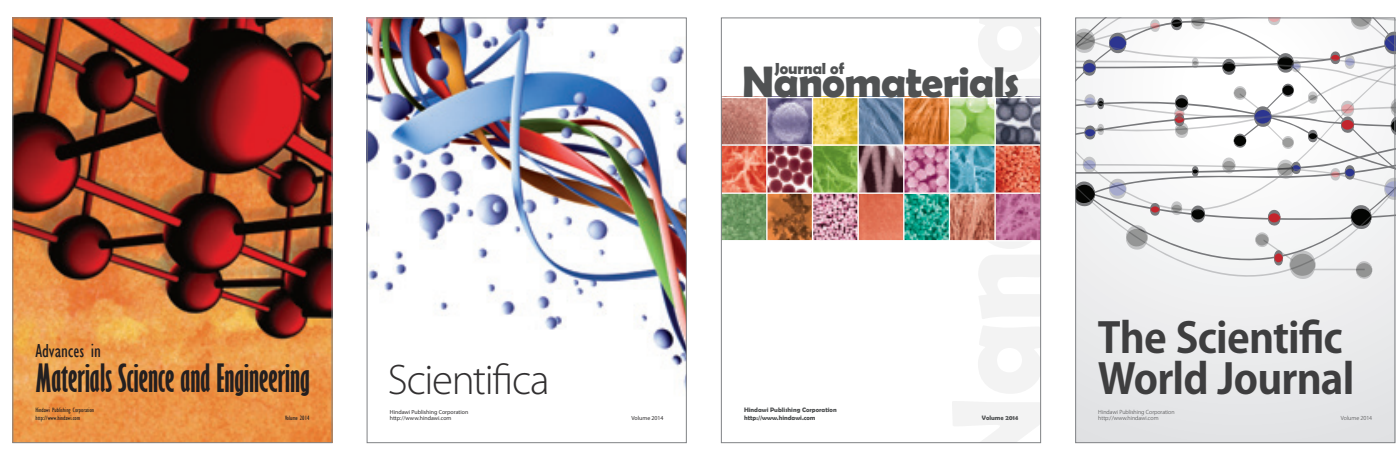

\section{The Scientific World Journal}
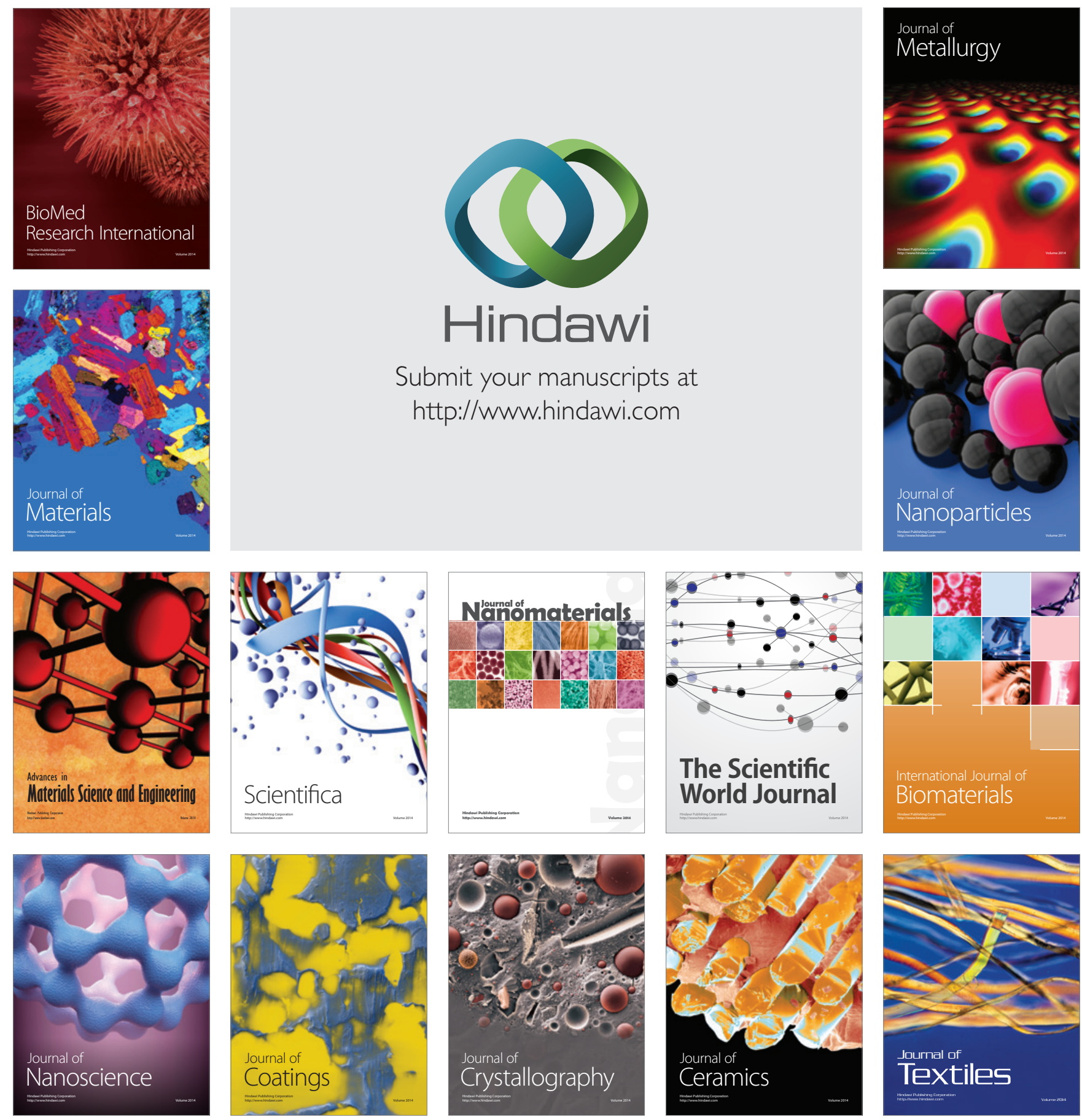